\title{
Autologous Serum Skin Test for Initial Identification of Auto- Antibodies in Chronic Urticaria
}

\author{
MOHAMMAD NURA ALAM, ${ }^{1}$ MOHAMMAD ALI, ${ }^{2}$ MOHAMMAD MONIRUZZAMAN KHAN, ${ }^{3}$ KEYA TARAFDER,${ }^{4}$ GOUTAM \\ KUMAR ACHERJYA, ${ }^{5}$ MUHAMMAD TANVIR MOHITH ${ }^{6}$
}

\begin{abstract}
Background: Autologous serum skin test (ASST) is easy to perform, cost effective measure for initial identification of auto-antibody in patients suffering from chronic urticaria which may occur due to autoimmune trigger by spontaneously developed auto-antibodies against $F c \mu R I$ receptor of skin mast cells.

Objective: This review is designed to see the positivity of ASST in patients suffering from chronic urticaria.

Materials \& Methods: This a retrospective data analysis conducted from the records of patients suffered from at least two episodes of urticaria or more in a week for 6 weeks and who did ASST aged more than 10 years between July 2015 to June 2017. These subjects were not pregnant or lactating mother, did not had urticarial vasculitis, predominant co-existing physical urticaria and had negative Hepatitis $B$ antigen and anti-nuclear antibody reports.

Results: Total 53 were included in this study of them 33 were female. Mean age was 32 years with a standard deviation (SD) of I I years. Among study patients 16 (30.2\% of total) had a positive ASST result. No age or sex difference was observed in positive ASST cases. In positive cases significant ( $\mathrm{P}$-value: $<0.00 \mathrm{I}$ ) mean induration difference of $2.75 \mathrm{~mm}$ with a $S D$ of $1.00 \mathrm{~mm}$ observed than induration produced by negative control.
\end{abstract}

Conclusion: ASST is easy and effective tool for initial identification of auto-reactive urticaria.

Key Words: autologous serum skin test, chronic urticaria, chronic spontaneous urticaria.

DOI: http://dx.doi.org//0.3329/jom.v19i2.37228

\section{Introduction:}

Urticaria is very common short-lived skin swellings due to transient leakage of plasma into the surrounding superficial dermis and its estimated lifetime prevalence is $20 \%{ }^{1}$ Release of numerous preformed chemotactic factors, enzymes,

1. Associate professor, Department of Dermatology and Venereology, International Medical College, Tongi, Gazipur, Bangladesh.

2. Assistant Professor, Haematology, National Institute of Cancer Research and Hospital, Mohakhali, Dhaka, Bangladesh.

3. Assistant Professor, Dermatology, Bangladesh Institute of Research \& Rehabilitation in Diabetes, Endocrine and Metabolic Disorders (BIRDEM), Dhaka, Bangladesh.

4. Lecturer, Department of Microbiology. Jessore Medical College \& Hospital, Jessore, Bangladesh.

5. Junior Consultant, Upazila Health Complex, Bagerpara, Jessore, Bangladesh.

6. Junior Consultant, Upazila Health Complex, Dakshin Surma, Sylht, Bangladesh.

Corresponding author: Mohammad Nura Alam, MBBS, DDV, Associate professor, Department of Dermatology and Venereology, International Medical College, Tongi, Gazipur, Bangladesh and consultant Dermatology, Farazy Hospital Limited, Banashree, Rampura, Dhaka, Bangladesh, e-mail: mnalamshopon@gmail.com, contact: +8801711315499.

Received: 18 January 2018;

Accepted: 19, April 2018 cytokines and proteoglycans from cutaneous mast cell granules or are newly generated from mast cell membrane mediates urticaria. Episodes of urticaria occurring at least twice a week for six weeks are considered to be chronic. More than $50 \%$ of the patients suffering from chronic urticaria $(\mathrm{CU})$ were diagnosed to have chronic spontaneous urticaria (CSU) as no cause could be identified. ${ }^{2}$ About one third of all cases of CSU are the result of an autoimmune trigger. Around 30-40\% of patients with CU spontaneously develop autoantibodies directed at the receptor Fc $\mu$ RI located on skin mast cells. ${ }^{3}$ Chronic stimulation of this receptor leads to chronic hives. Simultaneously patients may have other autoimmune conditions like autoimmune thyroiditis, celiac disease, type 1 diabetes, rheumatoid arthritis, Sjogren's syndrome or systemic lupus erythematosus. A subset of patients with $\mathrm{CU}$ has complement activating (C5a), histamine releasing $\mathrm{IgG}$ autoantibodies that degranulate mast cells and basophils by binding to the \pm -chain of the high-affinity $\mathrm{IgE}$ receptor. ${ }^{4}$

A clinical test to demonstrate circulat-ing endogenous whealinducing factors in urti-caria, the so-called autologous serum skin test (ASST), was developed in 1980..$^{5}$ Though Basophilic Histamine release assay is "Gold Standard" in detection of functional antibodies from serum of patients is time consuming and requires flowcytometry and standardization. Western blotting, enzyme-linked immunosorbent assay and 
flow cytometry using chi-merci cell lines expressing the human Fc $\mu \mathrm{RI} \pm$ useful for further identification and need validation. ${ }^{6}$ ASST has approximate sensitivity $70 \%$ with specificity $80 \%$ and indicates the presence of functional circulating autoantibodies against $\mathrm{Fc} \mu \mathrm{RI} \pm$ and/or $\mathrm{IgE}$, is the best in vivo clinical test in detection of histamine release activity in vitro when the positive result is defined as a pink, serum-induced wheal with a diameter e" $1.5 \mathrm{~mm}$ than adjacent wheal of normal saline control injection after 30 minutes. $^{7-8}$ ASST is easy to perform and need no costly instrument. In low resource country like Bangladesh, it may be an alternative for initial detection of functional antibodies in CSU patients and useful for consideration of immunomodulatory treatment. Till there is no data on ASST in Bangladesh, this retrospective database study was designed to see the positivity of ASST of CU patient in Bangladesh.

\section{Materials \& Methods:}

This is a retrospective data analysis of records of patents aged more than 10 years attended in Dermatology outpatient services of Farazy Hoapital limited, Block-E, Banosree, Dhaka, Bangladesh between July 2015 to June 2017 with history of more than 6 weeks urticaria or suspected urticaria occurring at least twice a week who did the ASST. Patients with urticarial vasculitis, predominant co-existing physical urticaria, patient who were pregnant or in lactation period and patients who were positive for Hepatitis B antigen and antinuclear antibody were excluded. Written informed consent was taken from every patient before performing ASST. Seven days before performing ASST all forms of antihistamines were discontinued and all forms of immunosuppressive drugs were stopped at least two months before performing ASST.

\section{Autologous serum skin test technique:}

Two milliliter venous blood was collected from antecubital vein of patients and placed in a sterile plastic tubes and allowed for clotting for 30 minutes at room temperature. After clotting serum was separated by centrifugation at $2000 \mathrm{rpm}$ for 15 minutes and kept in aliquots for use in the ASST. 0.05 milliliter autologous serum was injected intradermally into the volar aspect of forearm skin, avoiding the areas of wheeling within past 24 hours and equal volume $0.9 \%$ sterile saline (for negative control) were separately injected with $27 \mathrm{G}$ needles, leaving gaps of at least three centimeters. No positive control was used. Wheals and flare responses were measured after 30 minutes. A positive ASST was defined as a serum-induced wheal which was both red and had a diameter of $1.5 \mathrm{~mm}$ or more than the saline-induced response at 30 minutes ${ }^{7}$. No control patient was taken and no specificity or sensitivity was measured against any gold standard methods of identification of spontaneous antibody in patients of CSU.

\section{Statistical analysis:}

All statistical analyses were conducted using SPSS version 22 for windows with two-tailed $95 \%$ confidence interval. ASST data for positive and negative test results were analyzed for demographic data such as sex, mean age, related clinical indications. Continuous data is presented as mean, standard deviation (SD) and categorical data is presented percentages. $P$-value is extracted by using independent sample $t$-test for continuous data and chi-square test for categorical data.

\section{Results:}

Fifty-three ASST reports of chronic urticaria patients is analyzed who meet the inclusion criteria. Among them 20 $(37.7 \%)$ were female and rest were male. Male, female ratio was 1.65 and mean age 32 years with a SD of 11 years. (Table-1).

Table-1

Characteristics of ASST positive and ASST negative patients.

\begin{tabular}{lcccc}
\hline Characteristics & $\begin{array}{c}\text { All patients, } \\
\mathrm{n}=53 \\
(100 \%)\end{array}$ & $\begin{array}{c}\text { Positive } \\
\text { ASST, } \\
\mathrm{n}=16(30.2 \%)\end{array}$ & $\begin{array}{c}\text { Negative } \\
\text { ASST, } \\
\mathrm{n}=37(69.8)\end{array}$ & $\begin{array}{c}p \text { - } \\
\text { value }\end{array}$ \\
\hline Sex & $20(37.7)$ & $8(15.1)$ & $12(22.6)$ & $0.226^{\mathrm{ns}}$ \\
Male, $\mathrm{n}(\%)$ & $33(62.3)$ & $8(15.1)$ & $25(47.2)$ & \\
Female, $\mathrm{n}(\%)$ & $32 \pm 11$ & $34 \pm 10$ & $31 \pm 11$ & $0.471^{\mathrm{ns}}$ \\
Age in years, (mean $\pm \mathrm{SD})$ & & & & \\
Related clinical indications, $\mathrm{n}(\%)$ & $34(64.2)$ & $11(20.8)$ & $23(43.4)$ & $0.201^{\mathrm{ns}}$ \\
Chronic urticaria & $12(22.6)$ & $2(3.8)$ & $10(18.9)$ & \\
Atopic dermatitis with chronic urticaria & $1(1.9)$ & $0(0.0)$ & $1(1.9)$ & \\
Dermato-graphism with chronic urticaria & $1(1.9)$ & $0(0.0)$ & $1(1.9)$ & \\
Chronic urticaria with allergic rhinitis & $2(3.8)$ & $2(3.8)$ & $0(0.0)$ & \\
Psoriasis with atopy with chronic urticaria & $1(1.9)$ & $1(1.9)$ & $0(0.0)$ & $1(1.9)$ \\
Atopic dermatitis with chronic urticaria with dermato-graphism & $1(1.9)$ & $0(0.0)$ & $1(1.9)$ & \\
Infectious eczematoid dermatitis with suspected urticaria & $1(1.9)$ & $0(0.0)$ & \\
Seborrheic dermatitis with atopic dermatitis & &
\end{tabular}

$\mathrm{ASST}=$ autologous serum skin test, $\mathrm{n}=$ number of cases, $\mathrm{SD}=$ standard deviation, $\mathrm{ns}=$ not significant. 
Table-II

Test results of study patients $(n=53)$

\begin{tabular}{lccccccc}
\hline Interpretations in mm & Test result & $\mathrm{n}$ & Mean & \pm SD & Min- & Max & $p$-value \\
\hline Negative Control & Positive & 16 & 7.06 & \pm 1.73 & $3-$ & 9 & $0.009^{\mathrm{s}}$ \\
& Negative & 37 & 5.84 & \pm 1.41 & $3-$ & 8 & \\
Total & & 53 & 6.21 & \pm 1.60 & $3-$ & 9 & \\
Test induration & Positive & 16 & 9.81 & \pm 1.87 & $7-$ & 14 & $<0.001^{\mathrm{s}}$ \\
& Negative & 37 & 6.62 & \pm 1.36 & 4 & 9 & \\
Total & & 53 & 7.58 & \pm 2.12 & 4 & 14 & \\
Difference induration & Positive & 16 & 2.75 & \pm 1.00 & $2-$ & 5 & $<0.001^{\mathrm{s}}$ \\
& Negative & 37 & 1.00 & \pm 0.00 & $1-$ & 1 & \\
\hline Total & & 53 & 0.30 & \pm 0.46 & $1-$ & 5 & \\
\hline
\end{tabular}

$\mathrm{n}=$ number of cases, $\mathrm{SD}=$ standard deviation, $\mathrm{Min}=$ minimum, $\mathrm{Max}=$ maximum, $\mathrm{s}=$ significant

Sixteen patients of chronic urticaria were found positive ASST with significantly high $(p$-value: $<0.001)$ mean induration $9.81 \pm 1.87 \mathrm{~mm}$ and difference of induration $2.75 \pm 1.00 \mathrm{~mm}$ than negative control induration produced by $0.9 \%$ normal saline (table: II). All the patients experienced more than two episodes of urticaria in a week for six weeks among them 34 $(64.2 \%)$ had only chronic urticaria, $12(22.6 \%)$ patients had atopic dermatitis with urticaria and rest of the patients experienced dermato-graphism, allergic rhinitis, psoriasis, atopic dermatitis, infectious eczematoid dermatitis, seborrheic dermatitis along with chronic urticaria (Table 1).

Among 16 ASST positive patients 8 (15.1\%) patients were male and same number of patients were female and mean age of positive ASST patients were 34 years with SD of 10 years. No significant difference observed between male and female positive ASST cases. No significant difference also observed regarding age in ASST positivity (Table 1).

Eleven patients $(20.8 \%)$ out of 34 chronic urticaria patients, were found ASST positive. Two patients $(3.8 \%)$ of psoriasis with chronic urticaria were positive for ASST and no significant difference found among patients of different presentations (table: 1 ).

\section{Discussion:}

After introduction of ASST in 1986 several analyses done worldwide with a view to initial identification of auto-reactive antibody in CU patients and no previous study done in Bangladesh before. ${ }^{9}$ We found ASST positivity in this retrospective study of Bangladeshi population is $30.2 \%$. Sabroe et al. reported that one-third of their patients with CSU had circulating functional antibody against the high affinity $\mathrm{IgE}$ receptor $\mathrm{Fc} \mu \mathrm{RI} \pm$ or $\mathrm{IgE}^{7}{ }^{7}$ Nettis et al. reported that serum from 42 of 102 Italian patients with CSU (41.2\%) induced a wheal-and-flare response. ${ }^{1}$ Krause $\mathrm{K}$ et al. reported $24.7 \%$ ASST positivity in Thai patients. ${ }^{9}$ Caproni et al. showed that 23 of 68 Italian patients (34\%) with CSU had a positive reaction to ASST. ${ }^{10}$ A review of more than 50 worldwide different analysis of ASST positivity study by Krause $\mathrm{K}$ et al. showed mean positivity is $45.9 \%$ and positivity reported $35 \%-45 \%$ in most of the study among chronic spontaneous urticaria patients ${ }^{9}$. Godse K V showed $25 \%-45 \%$ patient of chronic urticaria had positive ASST. ${ }^{11}$ So, our findings in Bangladeshi population is more or less similar with other study.

Findings in terms of the female predominance of chronic urticaria is similar with Caproni et al. and Kulthanan $\mathrm{K}$ et al. ${ }^{10,12}$ But no significant sex difference observed ( $p$ value:0.226) among subjects with positive or negative ASST and also no significant age difference ( $p$-value:0.471) observed among positive or negative ASST subjects. These finding are similar to Krause $\mathrm{K}$ et al. findings. ${ }^{9}$

ASST is a recommended test of screening for identification of functional antibody in CU patients as ASST negativity excludes presence of functional antibodies in around $90 \%$ cases. ${ }^{13}$ So, from similar ASST positivity of this study with other studies, we recommend the use of this simple screening tool for initial detection of functional antibodies in Bangladesh.

However, in this study no comparison done regarding specificity or sensitivity with any gold standard methods of antibody detection and finally a multicenter comparative study of ASST with Basophilic Histamine release assay involving larger number of subjects recommended in Bangladesh. 


\section{Conclusion:}

Autologous serum skin test is cost effective, easy and safe test to detect auto-reactive antibody against mast cell membrane receptor with good positivity rate compared with other studies. We can safely use ASST for initial screening to identify auto-reactive antibody in chronic urticaria patients of Bangladesh. We hope that this retrospective analysis report will encourage further studies and understanding of the factors implicated in the CU.

\section{Conflict of interest: None.}

\section{References:}

1. Nettis E, Pannofino A, D'Aprile C, Ferrannini A, Tursi A. Clinical and aetiological aspects in urticaria and angio-oedema. Br. J. Dermatol. 2003;148(3):501-506.

2. Urticaria. In: James W D, Berger T G, Elston D M, editors Andrews' Diseases of the Skin: Clinical Dermatology, $12^{\text {th }}$ ed. Philadelphia: Elsevier 2016:p146.

3. The Skin in Immune, Autoimmune, Autoinflammatory and Rheumatic Disorders. In: Wolff K, Johnson R A, Saavedra A P, Roh E K, editors. Fitzpatrick's Color Atlas and Synopsis of Clinical Dermatology, $7^{\text {th }}$ ed. Mc Grew Hill: New York, 2013:p.309.

4. Caproni M, Giomi B, Volpi W, Melani L, Schincaglia E, Macchia D, et al. Chronic idiopathic urticaria: infiltrating cells and related cytokines in autologous serum-induced wheals. Clin immunol 2005;114(3):284-92.

5. Grattan CE, Wallington TB, Warin RP, Kennedy CT, Lbradfield JW. A serological mediator in chronic idiopathic urticaria-a clinical, immunological and histological evaluation. British Journal of Dermatology. 1986;114(5):58390.

6. Grattan CE, Sabroe RA, Greaves MW. Chronic urticaria. J Am Acad Dermatol. 2002;46(5):645-57; quiz 657-60.

7. Sabroe RA, Grattan CE, Francis DM, Barr RM, Kobza Black A, Greaves MW. The autologous serum skin test: a screen-ing test for autoantibodies in chronic idiopathic urticaria. Br J Dermatol 1999;140:446-52.

8. Sabroe RA, Seed PT, Francis DM, Barr RM, Black AK, Greaves MW. Chronic idiopathic urticaria: comparison of the clinical features of patients with and without anti-Fc epsilon RI or anti-IgE autoantibodies. J Am Acad Dermatol 1999;40:443-50.

9. Krause K, Metz M, Magerl M, Maurer M. Prevalence and relevance of skin autoreactivity in chronic urticaria. Expert Review of Dermatology. 2009;4(6):655-63.

10. Caproni M, Volpi W, Giomi B, Cardinali C, Antiga E, Melani L, et al. Chronic idiopathic and chronic autoimmune urticaria: clinical and immunopathological features of 68 subjects. Acta Derm Venereol 2004;84:288-90.

11. Godse KV. Autologous serum skin test in chronic idiopathic urticaria. Indian Journal of Dermatology, Venereology and Leprology. 2004;70(5):283.

12. Kulthanan K, Jiamton S, Gorvanich T, Pinkaew S. Autologous Serum Skin Test in Chronic Idiopathic Urticaria: Prevalence, Cor-relation and Clinical Implications. Asian Pacific Journal of Allergy and Immunology. 2006;24(4):201.

13. Konstantinou GN, Asero R, Maurer M, Sabroe RA, Schmid Grendelmeier P, Grattan CE. EAACI/GA2LEN task force consensus report: the autologous serum skin test in urticaria. Allergy. 2009;64(9):1256-68. 